\title{
Uma experiência de análise de videoaulas baseada no comportamento de estudantes
}

\author{
Francisco Genivan Silva ${ }^{1,3}$, Alan Santana ${ }^{1}$, Isabel Nunes ${ }^{2}$, Thiago Reis da Silva ${ }^{4}$, \\ Eduardo H. S. Aranha ${ }^{1}$, \\ ${ }^{1}$ Programa de Pós-Graduação em Sistemas Computacionais - PPgSC/UFRN \\ ${ }^{2}$ Instituto Metrópole Digital - IMD/UFRN \\ ${ }^{3}$ Instituto Federal de Educação, Ciência e Tecnologia do Rio Grande do Norte - \\ IFRN/Campus Parelhas \\ ${ }^{4}$ Instituto Federal de Educação, Ciência e Tecnologia do Maranhão - IFMA/Campus \\ São João dos Patos

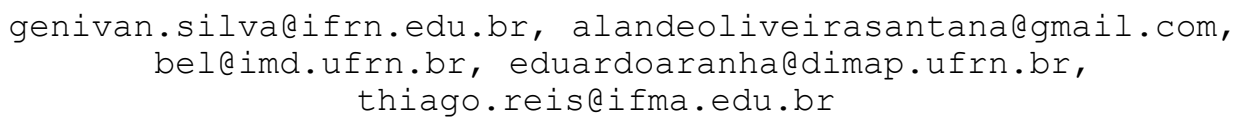

\begin{abstract}
Video lesson have been widely used in distance learning courses and the student behavioral pattern by watching them can help answer important questions about the quality of videotapes. However, this is an uncommon practice for most teachers, given the lack of knowledge about the subject. That said, the objective of this study is to systematize the analysis of student actions when watching videotapes, in order to facilitate the diffusion of this practice in the country's educational institutions. For this systematization, interviews, bibliographic surveys and video data analysis were carried out. The systematization was validated with its application in a video game programming course based on video lessons.
\end{abstract}

Resumo. As videoaulas têm sido bastante utilizadas em cursos à distância e o padrão de comportamento dos estudantes ao assisti-las pode ajudar a responder questões importantes sobre a qualidade das videoaulas. Porém, essa é uma prática pouco comum para a maioria dos professores, dada a falta de conhecimento sobre o assunto. Dito isto, o objetivo deste estudo é sistematizar a análise das ações de estudantes ao assistir videoaulas, visando facilitar a difusão dessa prática nas instituições de ensino do país. Para essa sistematização, foram realizadas entrevistas, levantamento bibliográfico e análise de dados de vídeo. A sistematização foi validada com a sua aplicação em um curso de programação de jogos digitais baseada em videoaulas.

\section{Introdução}

A explosão na oferta de cursos abertos em todos os níveis de ensino através da Internet, os chamados Cursos Online Abertos e Massivos (MOOC), tem demonstrado o potencial de ampliação no acesso à educação promovidos por esses ambientes de aprendizagem. Ao mesmo tempo, este fenômeno abre espaço para a criação de materiais didáticos sobre os mais variados assuntos e para o uso de diferentes mídias. Em especial, as videoaulas são muito utilizadas nos MOOCs e em cursos de ensino à distância (EAD).

Esta expansão no ensino e no uso de novas mídias, no entanto, traz consigo uma série de complicações e questionamentos acerca de sua real efetividade. Alguns 
VII Congresso Brasileiro de Informática na Educação (CBIE 2018)

Anais do XXIV Workshop de Informática na Escola (WIE 2018)

problemas das propostas educacionais que utilizam as videoaulas como recurso dizem respeito a dificuldade dos profissionais em empregar esta mídia como um recurso pedagógico. Segundo Serafim e Souza (2011, p.27), “o preparo dos docentes brasileiros para a utilização de mídias e objetos digitais como materiais didático-pedagógicos ainda é insipiente". Esta falta de preparo reflete na forma de condução da avaliação ou da não avaliação da qualidade dos materiais produzidos, e de como isto traz consequências para a aprendizagem dos estudantes.

Atualmente, não apenas as grandes plataformas de vídeo e de educação à distância utilizam videoaulas, mas, como lembrado por Barrére (2014), também há um número significativo de instituições de menor porte, instrutores e professores que empregam isolada ou sistematicamente este recurso. Apesar dessa popularização, há ainda uma carência de trabalhos que avaliem a qualidade das aulas baseado no comportamento dos alunos ao assisti-las. Neste contexto, o objetivo deste trabalho é investigar como sistematizar a análise do comportamento do estudante ao assistir videoaulas de forma a empoderar os gestores educacionais com informações relevantes para melhoria da qualidade das aulas.

\section{Análise de videoaulas}

A análise de vídeos de maneira geral não é uma atividade nova, mas nos últimos anos tem se diversificado e sido realizada sob diferentes ângulos e aspectos, utilizando métodos de coleta distintos e dados de diferentes fontes. Há, por exemplo, estudos que utilizam os chamados indicadores psicofisiológicos, como movimento dos olhos e expressões faciais [El Haddioui e Mohamed 2012; Cha e Kim 2016]. Estas técnicas, embora tenham se mostrado eficazes quanto ao dimensionamento, principalmente, dos níveis de atenção e concentração do espectador, são dispendiosas e complexas, pois exigem ambiente e equipamentos adequados para sua realização.

Um dos métodos mais aplicados para avaliação de videoaulas é a sondagem da opinião de estudantes e professores através da aplicação de questionários. Os trabalhos de [Silva et. al. 2015] e [Medeiros e Pansanato 2015] analisaram as respostas de estudantes e professores para apontar preferências de uso, formato e estilo das videoaulas, apresentando contribuições à produção de conteúdos e para o estudo das linguagens em vídeo.

Além destes trabalhos, há aqueles que se baseiam na análise de logs de interação dos usuários. Estes são estudos que visam extrair informações sobre as videoaulas a partir das interações dos usuários enquanto estes navegam no conteúdo disponibilizado. Com relação a estes estudos, podemos destacar os achados de Giannakos et. al., (2014), que criaram e utilizaram um sistema online para análise que se baseia no número de interações do usuário e que os relaciona com o desempenho dos estudantes.

Além deste trabalho, podemos citar também Brotherton e Abowd (2004), autores que propõem uma abordagem baseada no número de saltos (jumps) e no tempo de cada salto realizado na mídia para identificar perfis de estudantes. Já o estudo de Jadin et. al., (2009) utiliza capturas de tela para identificar ações de estudantes e assim classificar diferentes estratégias de aprendizagem. Há também estudos que analisam taxas de abandono e repetição de trechos das videoaulas [Kim et. al. 2014]. 
Todos esses trabalhos discutem a importância de conhecer como os estudantes interagem com as videoaulas a partir de arquivos de log. Apesar disso, pouco se observa em termos de aplicações práticas das análises propostas por esses trabalhos. Há assim uma aparente lacuna entre as pesquisas que estão sendo realizadas e o que é feito na prática. Este trabalho vem assim contribuir com a sociedade sistematizando a aplicação desses métodos de análise nas escolas.

\section{Metodologia}

A metodologia utilizada no desenvolvimento deste trabalho encontra-se organizada em duas etapas. A primeira é a de sistematização da análise dos dados de interação do aluno com as videoaulas. A segunda é a implementação desses mecanismos de análise e validação dos mesmos com sua aplicação prática em uma escola.

A etapa de sistematização é por sua vez organizada em duas partes. A primeira delas é a identificação de questões relevantes para serem respondidas durante a análise de videoaulas (Video Analytics). Para isso, foram realizadas entrevistas com professores que atuam com o desenvolvimento e aplicação de videoaulas. Já a segunda parte da etapa de sistematização tem como objetivo identificar mecanismos de análise que possam ser utilizados para responder as questões identificadas pelos professores entrevistados.

Uma vez definido como sistematizar a análise, a segunda etapa é a de validação dessa sistematização, na qual as seguintes atividades são aplicadas: implementação dos mecanismos de coleta e análise de dados sistematizados; aplicação dos mecanismos implementados em um caso real.

\section{Sistematização da análise}

Em nosso esforço para descobrir questões chave a respeito do comportamento dos estudantes em videoaulas, nós conduzimos uma entrevista semiestruturada com profissionais que utilizam e/ou criam conteúdos digitais. A entrevista foi realizada com 6 profissionais de educação, sendo 4 professores e dois profissionais que lidam com a criação de materiais didáticos. A entrevista se baseou em três eixos principais: contextualização do uso das videoaulas no processo de ensino; averiguação do conceito de qualidade de videoaula; e formas de avaliação de uma videoaula.

Sobre o uso de videoaulas no processo educacional, podemos destacar que todos os interlocutores reconhecem este recurso como parte fundamental dos cursos e disciplinas, sejam estes disponibilizados no ensino a distância ou em outras modalidades como no ensino híbrido.

Em suma, conseguimos extrair dessas entrevistas um conjunto de insights acerca da percepção destes profissionais quanto ao uso de videoaulas e o processo de avaliação de qualidade deste recurso. Podemos sintetizar as conclusões das entrevistas em 3 considerações principais: 1) é entendimento comum que as videoaulas são um recurso imprescindível na educação a distância ou presencial; 2) os professores de modo geral não avaliam as videoaulas que utilizam e desconhecem como fazer isso e 3) acreditam que conhecer o comportamento dos estudantes pode favorecer a aprendizagem. 
VII Congresso Brasileiro de Informática na Educação (CBIE 2018)

Anais do XXIV Workshop de Informática na Escola (WIE 2018)

\subsection{Identificação de questões relevantes}

Uma vez observada a visão dos entrevistados, foi possível definir um conjunto de questões para guiar a análise de videoaulas. Estas questões ajudam a compreender a forma como o comportamento dos estudantes pode ser utilizado como medida para avaliar sua relação com a aprendizagem ou a qualidade dos vídeos. A Tabela 1 apresenta as questões e as justificativas que embasam cada uma:

Tabela 1. Questões e justificativas.

\begin{tabular}{|l|l|}
\hline \multicolumn{1}{|c|}{ Questão } & \multicolumn{1}{c|}{ Justificativa } \\
\hline $\begin{array}{l}\text { Quais segmentos mais } \\
\text { vistos ou menos vistos no } \\
\text { vídeo? }\end{array}$ & $\begin{array}{l}\text { Identificar as partes menos assistidas de um vídeo ou os } \\
\text { picos de interação pode ajudar a destacar áreas de } \\
\text { desinteresse ou que são mais atraentes dos alunos. O que } \\
\text { pode indicar falhas metodológicas na apresentação do } \\
\text { conteúdo ou dificuldades de compreensão, por exemplo. }\end{array}$ \\
\hline $\begin{array}{l}\text { Há padrões diferentes de } \\
\text { visualização dos vídeos? }\end{array}$ & $\begin{array}{l}\text { Diferentes padrões geralmente indicam diferentes } \\
\text { estratégias de aprendizado, e conhecê-los pode ajudar a } \\
\text { melhorar o modo como as aulas em vídeo são criadas ou } \\
\text { personalizadas. }\end{array}$ \\
\hline $\begin{array}{l}\text { Qual o tempo total gasto } \\
\text { no vídeo? }\end{array}$ & $\begin{array}{l}\text { Investigar o tempo gasto no vídeo, além de sua duração, } \\
\text { pode contribuir na identificação da quantidade de esforço } \\
\text { necessário para consumir um determinado conteúdo. }\end{array}$ \\
\hline $\begin{array}{l}\text { Qual o momento do } \\
\text { abandono? }\end{array}$ & $\begin{array}{l}\text { O tempo de abandono é um bom indicador do interesse } \\
\text { dos alunos em uma videoaula. Conhecer este momento } \\
\text { pode contribuir na análise de engajamento com o recurso } \\
\text { ou com uma aula específica. }\end{array}$ \\
\hline
\end{tabular}

\subsection{Identificação de mecanismos de análise}

Esta etapa serviu para definir a estratégia a ser utilizada para responder as questões levantadas anteriormente. Conforme discutido no referencial teórico, há diversas opções de técnicas utilizadas para efetuar análise do uso de videoaulas, neste trabalho optamos por um mecanismo que pode facilmente ser acessado por professores e interessados em avaliar o comportamento de estudantes em ambientes educacionais. Este mecanismo é a coleta automática de dados realizada a partir da interação dos estudantes com o próprio sistema de informação.

Um protótipo para coleta de dados foi desenvolvido para ser utilizado em conjunto com sistemas web. Este protótipo utiliza parte da API do HTML5 que trata de vídeos e algumas funcionalidades foram desenvolvidas com a linguagem PHP. Este protótipo foi criado para capturar ações reais dos estudantes, enquanto utilizam o sistema, de modo particular, quando interagem no vídeo, como assistir, pausar ou avançar. Esses valores armazenados no arquivo de $\log$ incluem a identificação da videoaula específica, o tipo de ação que foi executada, a hora do dia em que as ações foram executadas, a duração de cada ação e a posição no vídeo em que foi executada.

A ideia é examinar os registros gerados por cada aluno para cada videoaula e extrair vários parâmetros como, por exemplo, o número de vezes que o botão play foi pressionado, o número de vezes que qualquer parte do vídeo foi revista e seguir com esta análise com outros parâmetros. 
VII Congresso Brasileiro de Informática na Educação (CBIE 2018)

Anais do XXIV Workshop de Informática na Escola (WIE 2018)

\section{Validação da sistematização proposta}

A proposta de análise desenhada foi efetivada com dados de um estudo realizado com 11 estudantes do $1^{\circ}$ ano do Ensino Médio de uma escola pública, sendo 9 do sexo masculino e 2 do sexo feminino. A seleção dos estudantes foi baseada em um convite realizado a estudantes dos turnos manhã e tarde que tivessem interesse em participar de uma pesquisa relacionada sobre o aprendizado do desenvolvimento de jogos digitais. Os alunos interagiram com um conjunto de 14 videoaulas e esses dados geraram o arquivo de $\log$ utilizado na análise.

\subsection{Implementação dos mecanismos de coleta e análise de dados}

O módulo de coleta de dados gerou um arquivo de log que posteriormente foi transformado em uma planilha eletrônica. A planilha continha 22064 linhas. Em uma análise inicial foi constatado que havia dados desnecessários e erros. Por essa razão, foi realizado um pré-processamento que envolveu a remoção manual de dados indesejados, campos vazios, dados errôneos, o que é conhecido como ruído. Esta etapa foi necessária para obtenção de um log de eventos consistente para a etapa de análise.

\subsection{Aplicação em curso baseado em videoaulas}

Para cada questão levantada durante a sistematização, apresentamos sua aplicação sobre os dados coletados durante o estudo com os estudantes do IFMA. Por causa da restrição de espaço, serão apresentados apenas exemplos que demonstram como cada análise foi efetuada e como os dados extraídos se relacionam com as características das videoaulas.

\subsubsection{Quais segmentos mais vistos ou menos vistos no vídeo?}

Os alunos tendem a consumir recursos educacionais de forma distinta, usando o play, pause ou ignorando o vídeo para aprender em seu próprio ritmo. A quantificação dessas atividades pode fornecer uma visão detalhada do aprendizado em MOOCs. Para fazer essa análise, podemos analisar o número total de vezes que os alunos assistiram a cada segundo no vídeo, ou seja, se o aluno repetidamente assistiu a mesma parte de um vídeo, cada segundo desse trecho foi considerado.

A Figura 1(a) e (b) utiliza como exemplo duas videoaulas distintas em termos de conteúdo e dificuldade. A videoaulas da Figura 1(a) trata de assunto introdutório do curso de programação para jogos e a videoaula da Figura 1(b) é de uma parte avançada do curso. Os gráficos apresentados nesta figura ilustram, principalmente, picos e declínios de visualização.

Nos gráficos apresentados, a linha principal (que varia) é referente ao somatório do número de vezes que todos os estudantes assistiram cada segundo no vídeo. Além desta linha há outras que servem de referência para esta variável, como o número máximo, o número mínimo e a média de visualizações do vídeo. É possível verificar que há picos de visualização nos trechos iniciais do vídeo e uma diminuição no seu terço final. Essa queda de visualizações não é surpresa e muitos estudos como o de Beatty, Merchant e Albert (2017) confirmam esta tendência.

Com relação aos picos, apesar de serem comuns no início dos dois vídeos, percebe-se que na videoaula da Figura 1(b), o número de visualizações se manteve acima da média por um tempo consideravelmente maior. Creditamos inicialmente esta distinção a diferença de conteúdo e dificuldade das aulas apresentadas, sendo a aula 
VII Congresso Brasileiro de Informática na Educação (CBIE 2018)

Anais do XXIV Workshop de Informática na Escola (WIE 2018)

mais avançada a que conseguiu manter mais a atenção dos estudantes. Para confirmar essa hipótese, também analisamos visualmente os trechos com picos de visualização e evidenciamos que um maior número de repetições sempre está relacionado a dois aspectos do vídeo: a realização de instruções práticas, normalmente ligadas ao conceito chave de cada vídeo; ou a apresentação de um conceito novo.
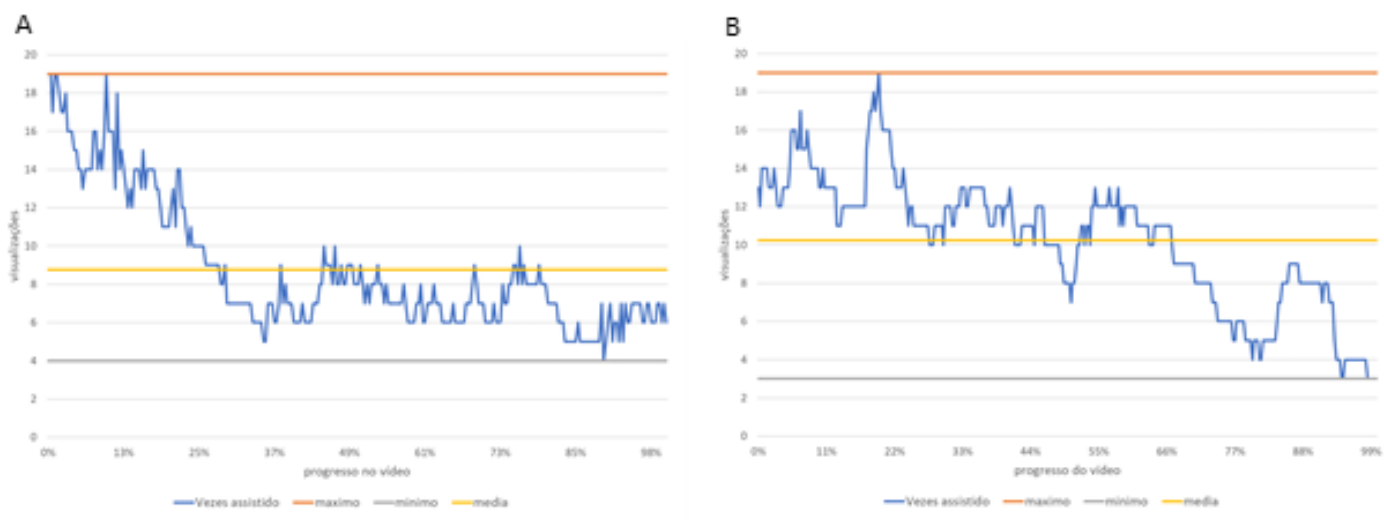

Figura 1. Número de visualizações em (a) aula introdutória e (b) aula avançada.

\subsubsection{Há padrões diferentes de visualização dos vídeos?}

Analisar a sequência de visualizações dos alunos pode ajudar a descobrir se existem padrões diferenciados de comportamento. Diferentes padrões geralmente indicam diferentes estratégias de aprendizado, e conhecê-los pode ajudar a melhorar o modo como as aulas em vídeo são criadas ou personalizadas. A sequência de visualização dos alunos é percebida a partir do refinamento do evento play nos vídeos, o que possibilita analisar a duração dessa ação e concentrar sequências mais curtas em uma maior.

Para responder esta pergunta, selecionamos dois alunos que apresentaram comportamentos de visualização nitidamente distintos entre si (Figuras 2 e 3). Mantivemos a análise para as videoaulas já citadas e apresentamos os gráficos e a análise dos padrões a seguir. Em ambas as figuras, cada barra representa uma seção de visualização contínua, um intervalo entre o play e o pause no log. Quando as barras (ou parte delas) se sobrepõem, significa que o trecho foi visualizado novamente em outro momento.

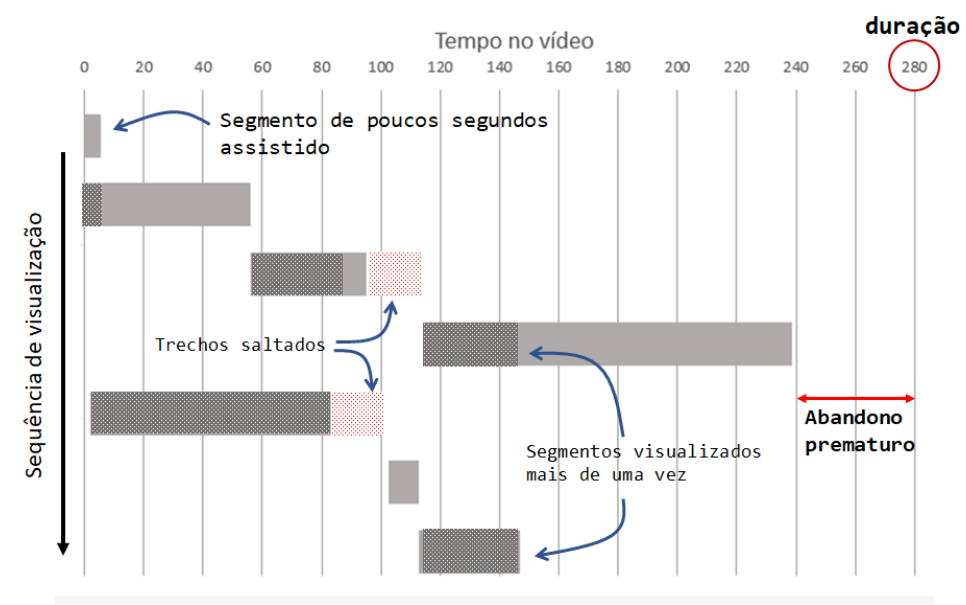

Figura 2. Sequência de visualização do estudante 1. 
VII Congresso Brasileiro de Informática na Educação (CBIE 2018)

Anais do XXIV Workshop de Informática na Escola (WIE 2018)

Na Figura 2, temos a exibição da sequência de trechos de vídeos visualizados pelo estudante 1. Sobre o comportamento dele, podemos identificar dois padrões de comportamento: saltos durante a execução e o retorno a trechos do vídeo, tanto para assistir as partes saltadas, quanto para repetir conteúdos já visualizados. Estes padrões de comportamento se diferenciam dos apresentados na Figura 3.

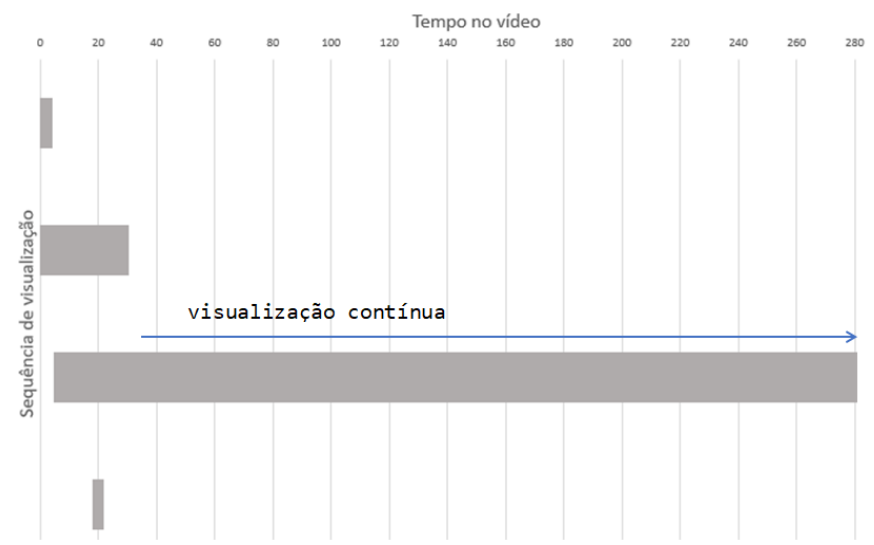

Figura 3. Sequência de visualização do estudante 2.

O gráfico da Figura 3 diz respeito ao comportamento de outro estudante no mesmo vídeo que o estudante 1 . Sobre este segundo estudante, fica clara a diferença de comportamento, sendo este caracterizado como visualização contínua e com poucas repetições. Conseguimos identificar esses mesmos dois padrões nos demais estudantes, o que torna possível agrupar estudantes através do comportamento e criar estratégias de ensino diferenciadas para cada grupo.

\subsubsection{Qual o tempo total gasto no vídeo?}

Para responder esta pergunta, apresentamos uma análise da distribuição dos valores de tempo total gasto pelo grupo de estudantes em cada videoaula (Figura 4). Para isso, fizemos o somatório dos tempos de visualização de cada segmento de vídeo assistido pelos estudantes. Além da distribuição dos dados, apresentamos como ponto de referência um indicador da duração de cada videoaula.

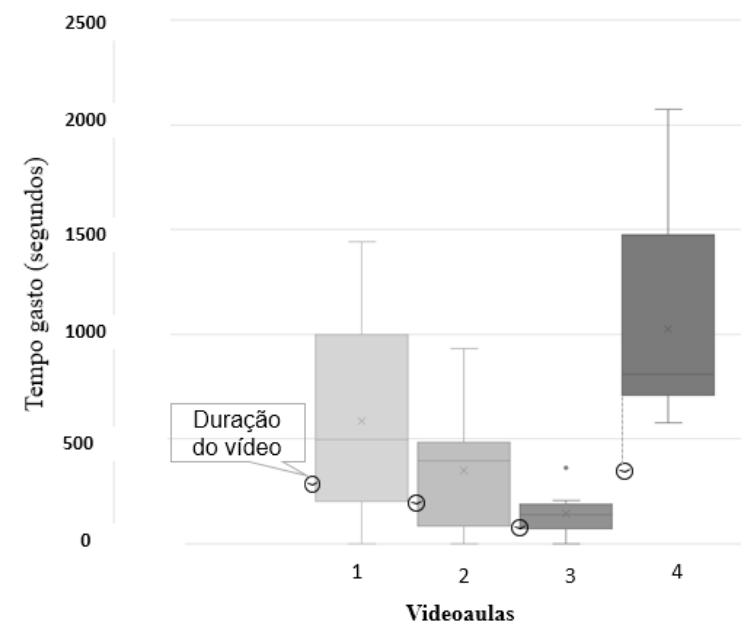

Figura 4: Tempo total gasto com as videoaulas. 
Quanto mais distantes do indicador de duração do vídeo estão o limite superior, a mediana e o terceiro quartil, maior é o esforço gasto pelos estudantes em um vídeo. Outra observação pode ser feita pelo tamanho dos box-plots. Vídeos mais longos costumam proporcionar uma maior dispersão dos alunos durante sua execução, além disso, tendem a causar um maior número de repetições dos trechos iniciais. Estas foram as razões encontradas para as diferenças de tempo entre os vídeos apresentados. Descartando os outliers, o vídeo mais longo fez com que os alunos permanecessem em média $288 \%$ mais tempo além de sua duração. Enquanto no vídeo mais curto este tempo foi de apenas 129\%, o que mostra que as diferenças de esforço necessárias para assimilar o conteúdo das aulas.

Além disso, trabalhos como os de Guo, Kim e Rubin (2014) e Kim et. al., (2014) mostram que o engajamento de estudantes diminui na proporção em que a duração da videoaula aumenta. Nós conseguimos confirmar esta hipótese ao analisar o percentual assistido de cada vídeo: os estudantes assistiram na média $86 \%$ do vídeo de menor duração, enquanto esse percentual ficou em $78 \%$ para o vídeo de maior duração.

\subsubsection{Qual o momento de abandono da videoaula?}

Um possível indicador do interesse dos alunos em uma aula em vídeo é o ponto em que ela é descartada. Na Figura 5, cada ponto é relativo a um aluno e o momento em que ele parou de assistir ao vídeo. Quanto mais tarde esse ponto, maior a evidência de que a aula conseguiu manter a atenção dos alunos por mais tempo. Para encontrar o ponto de abandono nas videoaulas, pode-se observar o último segundo visualizado pelo aluno, embora isto não indique que ele assistiu todo o vídeo até o abandono.

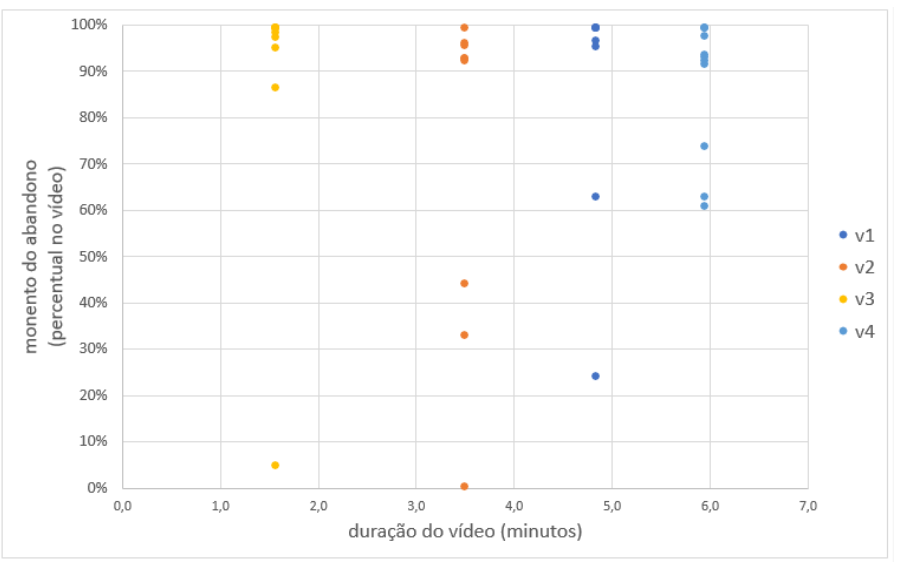

Figura 5: Pontos de abandono dos estudantes por vídeo.

O gráfico da Figura 5 mostra que o vídeo com menor duração é o que apresenta também menor dispersão e o que possui pontos de abandono mais próximos de seu final. $\mathrm{O}$ de maior duração apresenta uma taxa de dispersão maior ao se visualizar aproximadamente $60 \%$ do seu tempo de duração, sugerindo que este momento deve ser revisto no vídeo.

\section{Análise dos resultados}

À medida que as plataformas e serviços de ensino a distância se expandem, cresce também a quantidade de videoaulas e, consequentemente, o número de visualizações e 
VII Congresso Brasileiro de Informática na Educação (CBIE 2018)

Anais do XXIV Workshop de Informática na Escola (WIE 2018)

interações dos estudantes com este recurso. A literatura nos mostra que a análise dinâmica desses dados nos permite compreender melhor a experiência dos estudantes e como estes aprendem através das videoaulas.

Este trabalho não trata de sugerir técnicas ou mecanismos novos para análise de videoaulas, mas de apresentar uma abordagem procedimental baseada no sequenciamento de atividades que podem ser replicadas por professores e interessados em educação mediada por tecnologias. Dessa forma, estruturamos e validamos uma proposta de análise do comportamento dos estudantes em videoaulas de programação com o intuito de implementar um processo de melhoria deste recurso educacional. Além disso, nossos resultados apontam um conjunto de variáveis do comportamento de estudantes a partir do qual é possível direcionar ações que contribuem para o aperfeiçoamento das videoaulas e para a personalização do ensino.

A validação da proposta em uma escola conseguiu revelar alguns insights que podem ser utilizados por professores nas suas análises particulares: vídeos mais curtos tendem a garantir um maior engajamento; trechos que introduzem um conceito chave ou que apresentam uma nova ideia geram maior interesse; estudantes possuem padrões de interação que podem ser comuns, ajudando a agrupá-los.

Uma limitação percebida neste estudo foi a abordagem utilizada para a coleta de dados, dependente completamente da geração automática de dados da aplicação, sem a utilização de outros parâmetros como o feedback dos alunos. Além dessa limitação, uma outra diz respeito ao tamanho limitado da amostra: alunos e vídeos. Assim, os resultados encontrados aqui não podem (e não devem) ser generalizados. Apesar disso, este conjunto foi suficiente para demonstrar o potencial da análise de logs de interação com videoaulas para o processo de melhoria de qualidade dos vídeos (design instrucional) e da aprendizagem dos estudantes.

Para um entendimento mais abrangente do comportamento de estudantes e de modo a possibilitar um maior poder de generalização dos resultados, os seguintes estudos são sugeridos: aplicação da proposta em um número maior de cursos, abrangendo uma quantidade maior de alunos; investigação de outras questões relacionadas ao uso de videoaulas; a implementação completa de um sistema de análise automática e a investigação aprofundada da relação do comportamento dos estudantes no vídeo e a aprendizagem.

\section{Referências}

Barrére, E. (2014) Videoaulas: aspectos técnicos, pedagógicos, aplicações e bricolagem. $3^{\text {a }}$ Jornada de Atualização em Informática na Educação (JAIE 2014). $3^{\text {o }}$ Congresso Brasileiro de Informática na Educação (CBIE 2014).

Beatty, Brian J.; Merchant, Zahira e Albert, Michael. (2017) "Analysis of Student Use of Video in a Flipped Classroom". TechTrends. https://doi.org/10.1007/s11528-0170169-1

Brotherton, J.; Abowd, G. (2004) "Lessons learned from eclass: Assessing automated capture and access in the classroom". In: ACM Transactions on Computer-Human Interaction, vol 11, 2, ACM Press, pp. 121-155. 
VII Congresso Brasileiro de Informática na Educação (CBIE 2018)

Anais do XXIV Workshop de Informática na Escola (WIE 2018)

Cha, S.; Kim, W. (2016) “The Analysis of Learner's Concentration by Facial Expression Changes \& Movements". In International Journal of Applied Engineering Research ISSN 0973-4562 V.11, N.23, pp. 11344-11349.

El Haddioui, I.; Mohamed, K. (2012) "Learner Behavior Analysis through Eye Tracking". In International Journal of Computer Science Research and Application, V.2, I.2, pp. 11-18.

Giannakos, M. N.; Chorianopoulos, K.; Chrisochoides, N. (2014) "Collecting and Making Sense of Video Learning Analytics". In: IEEE Frontiers in Education Conference (FIE).

Jadin, T.; Gruber, A.; Batinic, B. (2009) "Learning with E-lectures: The Meaning of Learning Strategies," Educational Technology \& Society, vol 12, 3, pp. 282-288.

Kim, J.; Guo, P. J.; Seaton, D. T.; Mitros, P.; Gajos, K. Z.; Miller, R. C. (2014) "Understanding in-video dropouts and interaction peaks inonline lecture videos," Proc. of the first ACM conference on Learning@ Scale (L@S '14), ACM Press, pp. $31-40$.

Medeiros, S. F. L.; Pansanato, L. T. E. (2015) "Estudo das Preferências de Alunos e Professores sobre Videoaula para Identificar Requisitos de Software para Ferramentas de Produção". In: XXVI Simpósio Brasileiro de Informática na Educação (SBIE 2015).

Serafim, M.L.; Souza, R.P. (2011) "Multimídia na educação: o vídeo digital integrado ao contexto escolar". In. SOUSA, R.P., MIOTA, F.M.C.S.C., e CARVALHO, A.B.G., orgs. Tecnologias digitais na educação[online]. Campina Grande: EDUEPB. 276 p. ISBN 978-85-7879-065-3.

Silva, T. R.; Aranha, E. H. S.; Oliveira, W.; Fernandes, K. T.; Lucena, M. J. N. R. (2015) "Investigando dois formatos de videoaulas de programação de jogos digitais para alunos do ensino médio". In: XXI Workshop de Informática na Escola - WIE (2015), p.187-196. 